\title{
FIRST PRINCIPLES MODELING FOR THE CONTINUOUS HOT- PRESSING PROCESS OF MDF IN ECOSIMPRO
}

\author{
Ines Mostafa, Pedro Santos, Gloria Gutierrez, César de Prada \\ Instituto de Procesos Sostenibles, Dpto. Ingeniería de Sistemas y Automática, Escuela de Ingenierías Industriales, \\ Universidad de Valladolid. \\ C/ Real de Burgos s/n, 47011, Valladolid. enasgouda5@gmail.com, pedro.santos.bartolome@uva.es, \{gloria| \\ prada\}@autom.uva.es
}

\begin{abstract}
The continuous hot pressing of medium density fiberboard (MDF) is a common and efficient way to produce this popular wood-based panels used for furniture and construction. The goal of this work is to develop a first-principles model of the process based on previously available work, and to use it to formulate predictions by implementing its numerical resolution on the program EcosimPro by the methodology of finite volumes. Results of the model are studied and compared with previous results available for a similar model of the same process from previous studies. The final purpose of the simulation is to be implemented as an online simulation as part of an integrated optimization and control scheme for a real MDF plant.
\end{abstract}

Keywords: MDF, EcosimPro, continuous pressing.

\section{INTRODUCTION}

Medium density fiberboard (MDF) is a construction material produced from wood: during its production process the wood is chipped, softened with steam, grinded and mixed with adhesives, after which it undergoes a drying operation. Subsequent to this drying, the remaining fibers are compacted into an MDF panel, through a pressing process. Of particular importance due to its benefits is the particular case of continuous pressing.

Continuous pressing is, as is usually the case, more beneficial than batch pressing, by increasing efficiency due to removing the start-up and finishing times, with all associated costs in time, adjustment, and material. The press is the most expensive piece of equipment, as well as a key limiting factor of the production of the plant [2][4].

During compression, the wood is heated, which results in evaporation of bound water, as well as in a polycondensation reaction affecting the resins added to the wood in previous steps. The rheology of the mat changes significantly with this processes as well. The processes interact strongly with each other, requiring good knowledge of the fundamental mechanisms in order to obtain an accurate model.
A number of mathematical models have been presented in the literature to model the process of MDF hot pressing since the eighties, when the technology started its mainstream use. One of the first models was developed by Humphrey and Bolton (1989) [8], for the batch process based on heat and mass transfer, and further work has provided other models for the batch process since then. However, the evolution of the technology to the continuous process has led to new models, such as the one developed by Thoemen and Humphrey, (1999, 2001, 2003) [5][6][7], accounting for heat and mass transfer as well as rheological and adhesion mechanisms in a moving reference. Pereira, Carvalho and Costa (2006) have presented a model for the continuous pressing of MDF as well, based on a fixed reference frame [2].

The paper is structured as follows. Next, a brief description of the pressing process. Section 3 describes the model itself, based on the conservation laws of the fundamental mechanisms of mass and energy transfer occurring, as well as the assumptions used to simplify the model, along with further specifications of the process included in the boundary conditions. Section 4 gives the specifics for the resolution of the model for a particular case: starting conditions, as well as the numeric resolution strategy used: finite volumes. Finally, Sections 5 and 6 provide the results of the simulation, and the final conclusions obtained respectively.

\section{PROCESS DESCRIPTION}

The wood chips originating from a previous process accumulate on top of a transport belt, forming the wood mat, which moves at the constant speed of the transport belt in the $\mathrm{x}$ axis $v_{\text {mat }}$. It remains in contact with the environment for 2 meters, after which it reaches contact with the steel belt, which compresses it from its initial height $L$ to the press height. The steel belt is at a higher temperature, intended to dry and evaporate the water in the wood, and is the only heat source of the process.

The bound water contained in the mat will begin to evaporate, mainly at the point of contact with the steel, driving an increase in the air pressure that will produce 
a pressure gradient with the interior of the mat, along with a gradient with the environment due both to this effect and to the compression of the wood. All physical phenomena will then suffer strong interactions until a final equilibrium, and no equilibrium is found before the mat exits the belt.

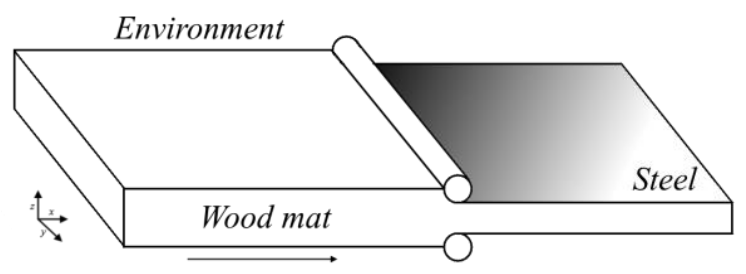

Figure 1: Macroscopic depiction of the continuous pressing process

\section{MODEL DEVELOPMENT}

The heat and mass transfer models published by Thomen and Humphrey (2003) [7] and later Pereira, Carvalho and Costa (2006) [2] have been adopted as the basis for further model development. The model is developed specifically for MDF and for continuous pressing.

\subsection{FORMULATION}

The model is formulated as the combination of three general conservation laws applied to any arbitrary volume of the mat. This formulation of the problem intuitively translates to the chosen resolution strategy by the finite volumes method.

\subsubsection{ENTHALPY BALANCE}

The total enthalpy accumulated in any volume of the mat is calculated as:

$$
\begin{gathered}
\frac{\partial H}{\partial t}=-\oint_{S} Q_{c o n d} d S-\oint_{S} Q_{c o n v} d S \\
+\Delta H_{v} \dot{m}_{e v}
\end{gathered}
$$

The integration over $S$ refers to the surface enveloping the volume.

In this same equation, $H$ is the total enthalpy of the volume, calculated as

$$
H=m_{g} C_{p g} T+m_{w} C_{p w} T
$$

Where $m_{g}$ and $m_{w}$ are the mass of gas and wood, respectively, and $C_{p g}$ and $C_{p w}$ are the specific heat of gas and wood. $T$ is the temperature of the element. In equation $1, Q_{\text {cond }}$ represents the heat flow by conduction through the surface, calculated by Fourier's law as

$$
Q_{\text {cond }}=-k \frac{d T}{d i}
$$

Where $i$ is the direction perpendicular to the surface $S$ and $k$ is the conductivity.
Similarly, $Q_{\text {conv }}$ represents the heat flow due to convection, calculated as:

$$
Q_{\text {conv }}=F C_{p g} T_{o}
$$

Where $F$ is the flow of air carrying the heat, and $T_{o}$ is the temperature of the air carried by this flow.

Finally, $\Delta H_{v}$ is the enthalpy of evaporation of water, and $\dot{m}_{e v}$ is the speed at which the mass is evaporating.

\subsubsection{GAS MASS BALANCE}

The evolution of the total gas mass accumulated in any volume of the mat is obtained as:

$$
\frac{\partial m_{g}}{\partial t}=-\oint_{S} F d S+\dot{m}_{e v}
$$

Where $F$ represents the flows of gas inside the wood. As the wood is a porous material, these can be calculated with Darcy's law

$$
F=\frac{\kappa}{\mu} \frac{d P}{d i}
$$

Where $\kappa$ is the permeability of the wood, $\mu$ the viscosity of the gas, and $P$ the total gas pressure, assumed to follow the ideal gas law such that

$$
P V \varepsilon=\frac{m_{g}}{M_{w}} R T
$$

Where $V$ is the volume, $\varepsilon$ the porosity, $M_{w}$ the molecular wheight of the gas, and $R$ the ideal gas constant.

\subsubsection{BOUND WATER MASS BALANCE}

The evolution of the total bound water mass accumulated in any volume of the mat is obtained by:

$$
\frac{\partial m_{b}}{\partial t}=-\oint_{S} J d S-\dot{m}_{e v}
$$

Where $J$ represents the diffusive flows of water in the wood, following Fick's law:

$$
J=D \frac{d u}{d i}
$$

Where $\mathrm{D}$ is the diffusion coefficient, which may be obtained by the formula provided by Siau (1984) [1][3]

$$
\begin{aligned}
& D_{m} \\
& =\frac{0.07}{\left(1-\varepsilon^{2}\right)(1-\varepsilon)} \exp \left(-\frac{9200-70 u}{R T}\right)
\end{aligned}
$$

And $\mathrm{u}$ is the bound water percentage.

\subsection{MODELLING ASSUMPTIONS}

In order to simplify the development model, some assumptions are included:

1. Water vapor content does not significantly influence the properties of the gas.

2. Diffusion of water vapor in the gas phase is negligible. 
3. Water evaporates completely when in contact with the steel belt.

4. Evaporation of water inside the mat is insignificant.

5. The steel belt presents no resistance to heat transfer and is at a constant temperature $T_{\text {steel }}$.

6. The environment presents no resistance to heat or mass transfer, and is at constant properties $P_{a m b}, T_{a m b}$

\subsection{BOUNDARY CONDITIONS}

Three boundary conditions are required at the surface of the mat, one for each of the conservation laws. The mat surface can itself be divided in two sections: one in contact with the environment, and the other in contact with the steel, leading to the following conditions:

Enthalpy balance:

- Environment: $T=T_{a m b}$

- $\quad$ Steel: $T=T_{\text {steel }}$

Gas mass balance:

- Environment: $P=P_{a m b}$

- $\quad$ Steel: $F=0$

Bound water mass balance:

- Environment: $J=0$

- Steel: $u=0$

All surfaces are in contact with the environment within the first 2 meters, after which the limiting surfaces in the $\mathrm{z}$ axis enter in contact with the steel.

In order to reduce the required computational power, there is an incentive to reduce the volume to be studied. For current study, a symmetry condition is imposed on the mat, so that symmetry planes divide the mat in both the $\mathrm{z}$ and $\mathrm{y}$ directions: Thus, the mat is divided in 4 symmetric pieces, and only one of this must be studied for our purposes, reducing the number of variables and equations to one quarter of the original.

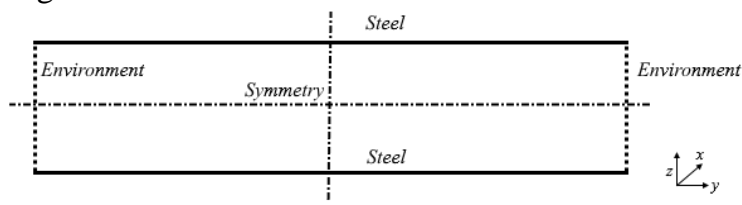

Figure 2: Boundary conditions of the problem after contact with the steel

Special boundary conditions can be included for the surfaces in contact with a symmetry plane. This are simple: where a surface is contained in a symmetry plane, it is true that any flow must have a symmetric opposite, so that total flow $f=0$. Therefore:

- $F=0$

- $Q_{\text {cond }}=0$

- $J=0$

\section{MODEL RESOLUTION}

The model is implemented in EcosimPro, a commercial modelling and simulation tool. Because EcosimPro does not require information about the causality in the model, both the editing of boundary or initial conditions and the further updating of the model are reduced in complexity.

Because all the MDF is moving at a constant speed $v_{\text {mat }}$, the system is studied using a moving reference system anchored to a point of the mat situated at $(0,0,0)$ at $t=0$. For the current study includes only the evolution of a single "slice" of mat at stationary conditions are studied, however, it is of note that, dynamic conditions can be easily included by adding additional segments of the mat, "before" and "after".

Because the process is studied at stationary conditions and from a moving reference system, the $\mathrm{x}$ axis is in practice replaced by the equivalency $x=v_{\text {mat }} * t$.

\subsection{INITIAL CONDITIONS}

Starting conditions are provided for the properties of the mat. For the current study, the properties of the MDF chips that form the mat are assumed to be homogeneous at the start of the process, but heterogeneous composition of raw material could be easily studied with the model.

\begin{tabular}{|l|l|}
\hline Operating conditions & Mat characteristics \\
\hline Pressing speed: & Temperature: $\mathrm{T}_{\mathrm{i}}=40^{\circ} \mathrm{C}$ \\
vcx $=0.129 \mathrm{~m} /$ & Gas pressure: \\
Press length: $28 \mathrm{~m}$ & $\mathrm{P}_{\mathrm{go}}=101.325 \mathrm{kPa}$ \\
Maximum & Moisture content: \\
temperature in the & $\mathrm{u}_{\mathrm{i}}=11 \%$ \\
steel belt: $\mathrm{T}_{\text {pmax }}=200^{\circ} \mathrm{C}$ & Density: $\boldsymbol{\rho}_{\text {co }}=100 \mathrm{~kg} / \mathrm{m}^{3}$ \\
Ambient temperature: & \\
$\mathrm{T}_{\mathrm{a}}=25^{\circ} \mathrm{C}$ & \\
Ambient pressure: & \\
$\mathrm{P}_{\mathrm{a}}=101.325 \mathrm{kPa}$ & \\
\hline
\end{tabular}

Table 1: initial operating conditions and mat characteristics to be used in the simulation

\subsection{FINITE VOLUMES METHOD}

In order to permit the numerical resolution of the equations, they have to be reduced from PDEs to DAEs, which EcosimPro can handle through included solvers. As the equations are already formulated as the conditions on any arbitrary volume, the use of the finite volumes strategy is easily implemented by the 
assumption that the mat can be divided into a number of finite volumes with homogeneous properties.

This methodology presents great advantages such as its intuitiveness for engineering processes such as this, and the fact that the conservation laws are strictly followed.

The mat is divided into elements of length $\Delta y$ and $\Delta z$ for the $\mathrm{y}$ and $\mathrm{z}$ azis respectively. This results in two axis that will go through discrete values from 1 to $2 *$ $N y$, and $2 * N z$. As symmetry conditions have been included in the boundary conditions, we need only study one fourth of the matress, and so the elements will range from $(0,0)$ to $(\mathrm{Ny}, \mathrm{Nz})$. The length of element $\mathrm{x}$ is determined by the size used by EcosimPro, which changes dynamically.
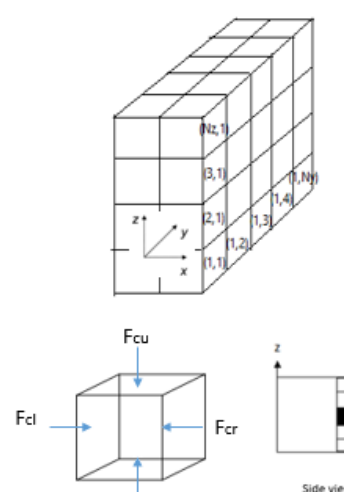

Fed

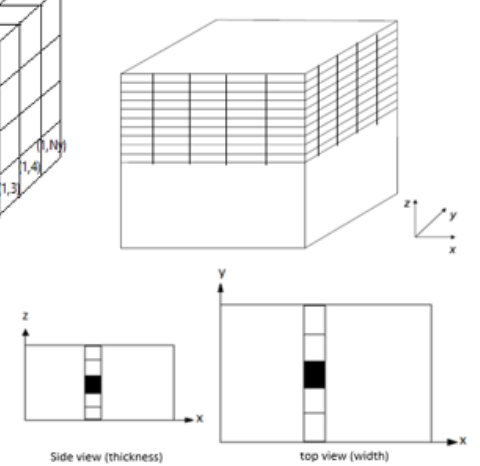

Side view (thickness)

rically divided into two

Figure 3: the mat is symmetrically divided into two halves and the upper one is divided into a number of finite volumes for simulation.

Where it would be normally necessary, $\Delta x$ can be replaced with $\frac{d x}{d t}=v_{\text {mat }}$.

For this case, $N y=5, N z=5$.

The volumes are described as polyhedrons, using in this case cuboids. This allows for the transformation of the surface integrals to a summation:

$$
\oint_{S} f d S=\sum_{j} f * A_{j}
$$

Where $j$ is the numeration of the faces, and $A_{j}$ is the area of that face, easily calculated as the product of the lengths of its sides.

\section{RESULTS \& DISCUSSION}

The following section shows the results of the simulation with the model with the proposed initial conditions and resolution method. Results are focused on the moisture, temperature and pressure profile along the $y$ axis, and compared with results for a similar process by Pereira, Carvalho and Costa 2006 [2] and Thoemen and Humprey [7].

\subsection{MOISTURE CONTENT PREDICTIONS}

The evolution of the moisture profile is shown in Figure 4. Physically consistent, as the elements in contact with the hot metal are the leading force of drying.

When contrasted with the results of [2] in Figure 5, it can be seen that, while the evolution of the variable shows similar trends, previous results predict both a faster drying in the surface, and a slower one in the interior of the wood. Clearly, the second effect is due to a lower diffusion parameter in Equation 9, while the first can be attributed to the inclusion of the effect of vaporization inside the wood, particularly significant at the edge, where the temperature is highest.

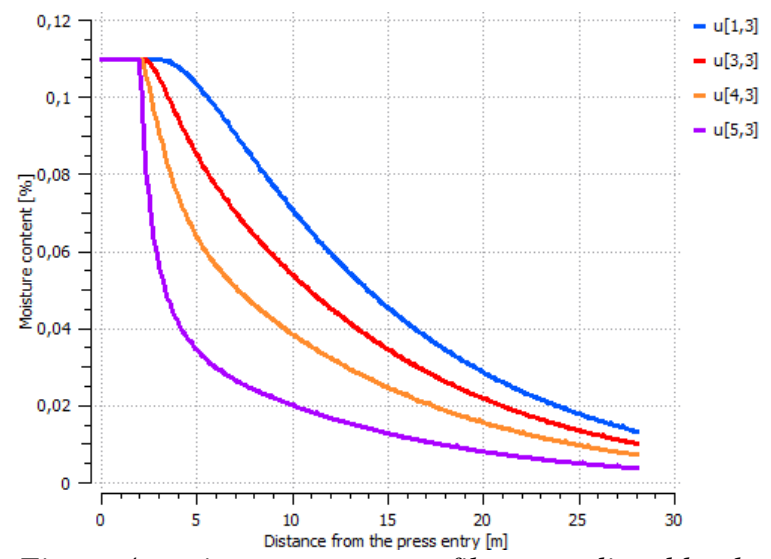

Figure 4: moisture content profile as predicted by the current model

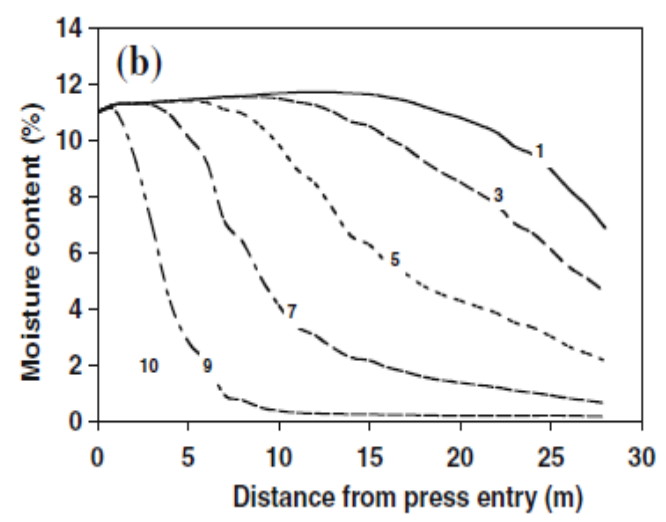

Figure 5: moisture content profile as presented by Pereira, Carvalho and Costa's (2006) moisture content profile

\subsection{TEMPERATURE PREDICTIONS}

The evolution of the temperature profile is shown in Figure 6. Physically consistent, as the elements in contact with the hot metal increase their temperature faster. 
When compared to previous results in Figure 7 however, very different temperature profiles are observed inside the wood mat, suffering an effect that dampens the increase in temperature. This effect is attributed to the inclusion of the evaporation of water inside de mat to thermodynamic equilibrium, which will include an additional cost to increasing temperature, as this requires an additional energy cost in evaporation. The reason why this effect is not seen in the surface layer is clear when the remarks of the previous subsection are taken into account: the surface layer quickly losses its bound water, and thus has no dampening of its heating.

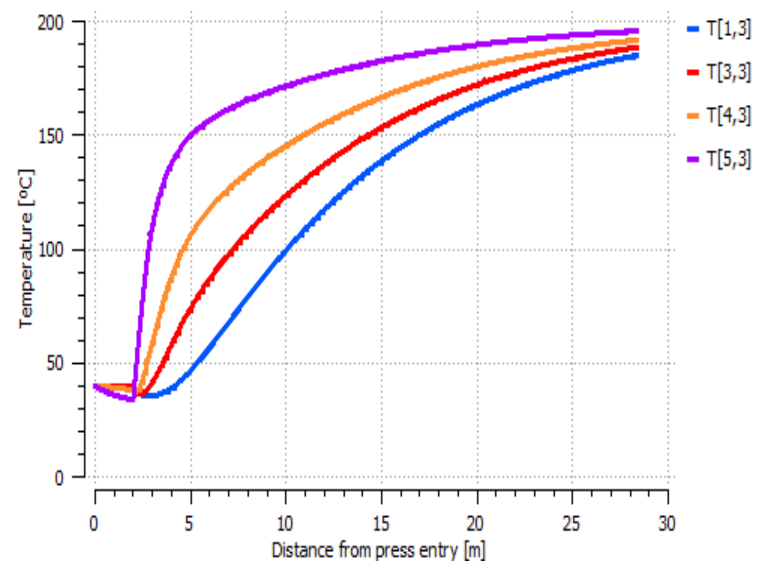

Figure 6: temperature profile as predicted by the current model

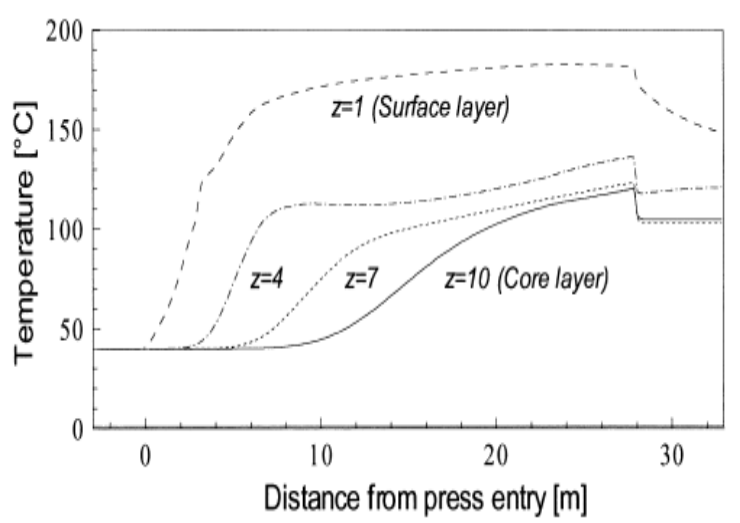

Figure 7: Temperature profile as predicted by Thoemen and Humphrey 2003 [7]

\subsection{GAS PRESSURE PREDICTIONS}

The evolution of the pressure profile is shown in Figure 8. The sudden peak after compression is due to the simulation of a single layer of wooden mat that advances in time in the direction $\mathrm{x}$, which prevents backflow in $\mathrm{x}$ which would smooth the shape of the peak. Further development of the simulation into a multilayer simulation in order to study transient developments will also remove this issue.

The comparison with Figure 9 shows two main differences: First, gas pressure descends much more rapidly in Figure 8: This is attributed to a higher value of the permeability $\kappa$ in Equation 6. Additionally, it is seen that vapor pressure, not included in our simulation due to the simplification of the evaporation process, is expected to greatly influence the profile.

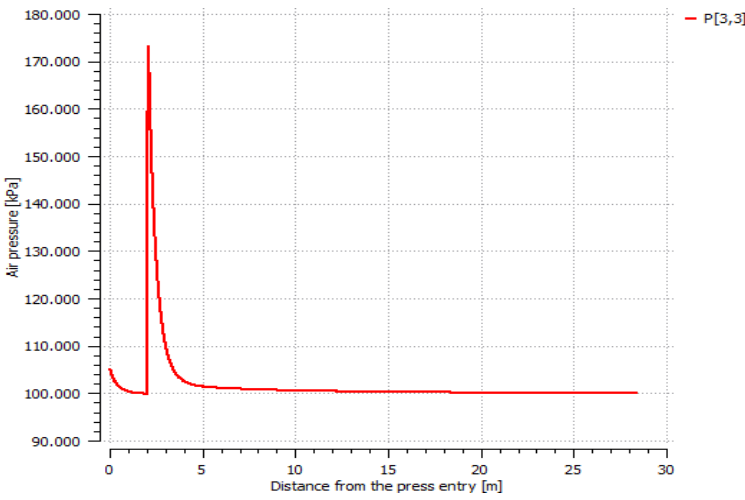

Figure 8: gas pressure profile as predicted by the model

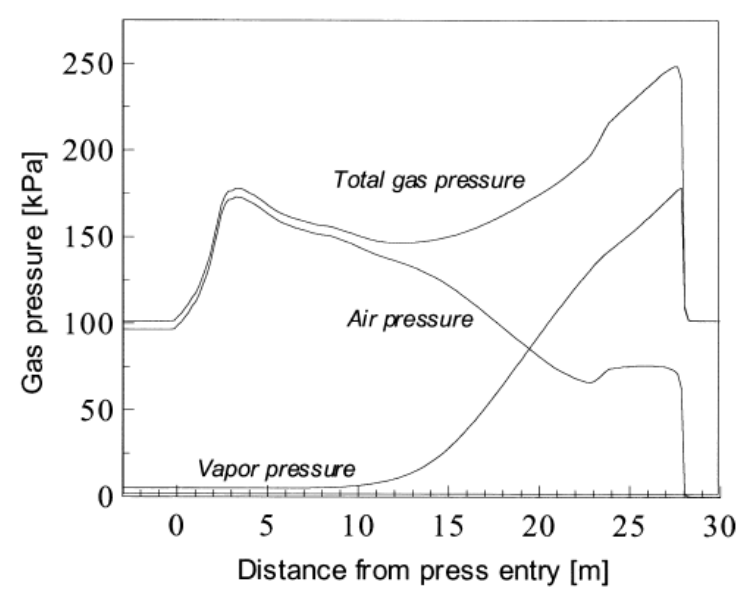

Figure 9: gas pressure profile as presented by Thoemen and Humphrey (2003) [7]

\section{CONCLUSION}

A model of wood mat drying under continuous pressing was developed by following conservation laws applied to an arbitrary volume, and successfully implemented in EcosimPro by extending this model to the well-known finite volume method. The evolution of temperature, moisture and pressure were particularly studied, and shown to have significant divergence with previous literature.

The evaporation mechanism is assumed to be simplified for first simulation (Assumptions 3 and 4 of Section 3.2). This simplification is, at first sight, valid: the humidity profile is similar, with differences caused mainly by the different diffusion coefficients.

However, the effects of this assumption is seen in the temperature and pressure profile: Thus, while the amount that evaporates inside the wood mat is 
negligible with respect to the amount that evaporates in direct contact with the metal (as assumed), it is seen that this small amount causes great interference with the profiles of other parameters inside the mat, such as temperature and pressure.

Further work includes the inclusion of the evaporation inside the wooden mat and a comparison of results, as well as the development of the simulation into a multilayer approach capable of simulating discontinuous operations such as startup.

\section{References}

[1] Gupta, A. (2007). Modelling and optimization of MDF hot pressing.

[2] Pereira, C., Carvalho, L. M., \& Costa, C. A. (2006). Modeling the continuous hot-pressing of MDF. Wood science and technology, 40(4), 308.

[3] Siau, J.F., (1984). Transport processes in wood. Syracuse Univ. Press, New York. 245pp.

[4] Sturgeon MG, Law ML (1989) Continuous pressing of medium density fiberboard at Nelson Pine Industries New Zealand. In: Proceedings of the third European Panel Products Symposium, Washington State University, Pullman, Washington, pp 179-185

[5] Thoemen H, Humphrey PE (1999) The continuous pressing process for wood-based panels: an analytical model. Proceedings of the third European Panel Products Symposium, Llandudno, Wales, UK, pp 18-30

[6] Thoemen H, Humphrey PE (2001) Hot-pressing of wood-based composites: selected aspects of physics investigated by means of simulation. In: Proceedings of fifth European Panel Products Symposium, Llandudno, North Wales, UK, pp 38-49

[7] Thoemen H, Humphrey PE (2003) Modeling the continuous pressing process for wood-based composites. Wood Fiber Sci 35:456-468

[8] Thoemen, H., \& Humphrey, P. E. (2006). Modeling the physical processes relevant during hot pressing of wood-based composites. Part I. Heat and mass transfer. Holz als Roh- und Werkstoff, 64(1), 1.

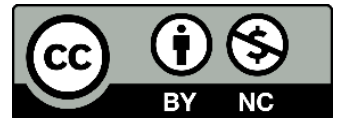

(C) 2018 by the authors. Submitted for possible open access publication under the terms and conditions of the Creative Commons Attribution CC-BY-NC $3.0 \quad$ license (https://creativecommons.org/licenses/by-nc/3.0). 